\title{
Dilepton production in elementary and in heavy ion reactions
}

\author{
Christian Fuchs and Amand Faessler \\ Institut für Theoretische Physik \\ Universität Tübingen, D-72076 Tübingen, Germany
}

\begin{abstract}
We present a unified description of the vector meson and dilepton production in elementary and in heavy ion reactions. The production of vector mesons $(\rho, \omega, \phi)$ is described via the excitation of nucleon resonances $(R)$. The theoretical framework is an extended vector meson dominance model (eVMD) for resonance decays $R \longmapsto N V$ with arbitrary spin which is covariant and kinematically complete. The eVMD includes thereby excited vector meson states in the transition form factors. The model has successfully been applied to $\omega$ and $\phi$ production in $p+p$ reactions. The same model is used to describe the dilepton production in elementary reactions where corresponding data are well reproduced. However, when the model is applied to heavy ion reactions in the BEVALAC/SIS energy range the experimental dilepton spectra measured by the DLS Collaboration are significantly underestimated at small invariant masses. In view of this fact we discuss further medium effects: One is a substantial collisional broadening of the $\rho$ and in particular of the $\omega$ meson in the vicinity of the $\rho / \omega$-peak. The second medium effect is the destruction of quantum interference in a dense medium. A decoherent dilepton emission through vector mesons decays enhances the corresponding low mass dilepton yield in heavy ion reactions and improves the agreement with existing data.
\end{abstract}

\section{Introduction}

One of the important questions which theorists face at present is the dependence of hadron properties on medium effects. Medium effects manifest themselves in the modification of widths and masses of resonances produced in nuclear collisions. The magnitude of such changes depends thereby on the density and the temperature of the medium. E.g., the proposed Brown-Rho scaling [1] is equivalent to a reduction of the vector meson masses in the nuclear medium. The same conclusion is obtained from QCD sum rules 2] and within effective hadronic models 3]. The dispersion analysis of forward scattering amplitudes [4, 5, 6, 7] showed that vector meson mass shifts are in general small and positive, whereas at low momenta they can change the sign which is in qualitative agreement with the Brown-Rho scaling and the results from QCD sum rules. However, the question of in-medium masses must finally be settled experimentally.

Dilepton spectra from heavy-ion collisions are considered as a suitable tool for this purpose. The CERES [8] and HELIOS 9] Collaborations measured dilepton spectra at CERN and found a significant enhancement of the low-energy dilepton yield below the $\rho$ and $\omega$ peaks $[8$ in heavy reaction systems $(P b+A u)$ compared to light systems $(S+W)$ and proton induced reactions $(p+B e)$. Theoretically, this enhancement can be explained within a hadronic picture by the assumption of a dropping $\rho$ mass [10] or by the inclusion of in-medium spectral functions for the vector mesons [11, 12. In both cases the enhanced low energetic dilepton yield is not simply caused by a shift of the $\rho$ and $\omega$ peaks in the nuclear medium but it originates to most extent from an enhanced contribution of the $\pi^{+} \pi^{-}$annihilation 
channel which, assuming vector dominance, runs over an intermediate $\rho$ meson. An alternative scenario could be the formation of a quark-gluon plasma which leads to additional $(p Q C D)$ contributions to the dilepton spectrum [11, 13.

A similar situation occurs at a completely different energy scale, namely around $1 \mathrm{~A} . \mathrm{GeV}$ incident energies where the low mass region of dilepton spectra are underestimated by present transport calculations compared to $p p$ and $p d$ reactions. The corresponding data were obtained by the DLS Collaboration at the BEVALAC 14. However, in contrast to ultra-relativistic reactions (SPS) the situation does not improve when full spectral functions and/or a dropping mass of the vector mesons are taken into account [15, 16, 12. This fact is known as the DLS puzzle. The reason lies in the fact that both, possible $p Q C D$ contributions as well as a sufficient amount of $\pi^{+} \pi^{-}$annihilation processes are absent at intermediate energies. Also a dropping $\eta$ mass can be excluded as a possible explanation of the DLS puzzle since it would contradict $m_{T}$ scaling 12. Furthermore, chiral perturbation theory predicts only very small modifications of the in-medium $\eta$ mass [17. Thus one has to search for other sources which could explain the low mass dilepton excess seen in heavy ion reactions. Dilepton spectra were also measured at KEK in $p+A$ reactions at a beam energy of $12 \mathrm{GeV}$ [18. Also here an excess of dileptons compared to the known sources was observed below the $\rho$-meson peak and interpreted as a change of the vector meson spectral functions. These data were recently analyzed in Ref. [19], again without success to explain the experimental spectrum within a dropping mass scenario and/or by a significant collision broadening of the vector mesons. Since the vector meson peaks are not resolved experimentally [14, the problem to extract in-medium masses directly from experimental data remains extremely difficult.

For all these studies a precise and rather complete knowledge of the relative weights for existing decay channels is indispensable in order to draw reliable conclusions from dilepton spectra. In [20] a systematic study of meson decay channels was performed, including channels which have been neglected so far, such as e.g. four-body decays $\rho^{0} \rightarrow \pi^{0} \pi^{0} e^{+} e^{-}$. However, as has been shown in [21] in $p p$ reactions the contributions of these more exotic channels are not large enough to enhance the low mass dilepton yield at incident energies around $1 \mathrm{AGeV}$. Here the low mass dilepton spectrum is dominated by the $\eta$ and the contributions from the decay of baryonic resonances [15, 21, 22].

The importance of the resonance contribution to the dilepton yield in elementary and heavy ion reactions has been stressed in several works [21, 23, 24, 25, 26, 27, 28, 29, 30, 31, 32, 33. In [33] we calculated in a fully relativistic treatment of the dilepton decays $R \rightarrow N e^{+} e^{-}$of nucleon resonances with masses below $2 \mathrm{GeV}$. Kinematically complete phenomenological expressions for the dilepton decays of resonances with arbitrary spin and parity, parameterized in terms of the magnetic, electric, and Coulomb transition form factors and numerical estimates for the dilepton spectra and branching ratios of the nucleon resonances were given. In 21] this approach was applied to the dilepton production in $p p$ reactions at BEVALAC energies. The relevant elementary hadronic reactions were systematically discussed. It is demonstrated that the resonance model provides an accurate description of exclusive vector meson production in nucleon-nucleon collisions $N N \rightarrow N N \rho(\omega)$ as well as in pion scattering $\pi N \rightarrow N \rho(\omega)$. The resonance model allows further to determine the isotopic channels of the $N N \rightarrow$ $N N \rho(\omega)$ cross section where no data are available. As discussed in 34, a peculiar role plays thereby the $N^{*}(1535)$ resonance which, fitting available photo-production data, has a strong coupling to the $N \omega$ channel. Close to threshold this can lead to strong off-shell contributions to the $\omega$ production cross section 34] which are also reflected in the dilepton yields.

The reaction dynamics of heavy ion collisions is described within the QMD transport model [35] which has been extended, i.e. the complete set of baryonic resonances $\left(\Delta\right.$ and $\left.N^{*}\right)$ with masses below $2 \mathrm{GeV}$ has been included in the Tübingen transport code. One purpose of the present investigations is to extract information on the in-medium $\rho$ - and $\omega$-meson widths directly form the BEVALAC data [14. The dilepton spectra, distinct from the vector meson masses, are very sensitive to the vector meson in-medium widths, especially the $\omega$-meson. The collision broadening is a universal mechanism to increase particle widths in the medium. E.g., data on the total photo-absorption cross section on heavy 
nuclei [36] provide evidence for a broadening of nucleon resonances in a nuclear medium 37. The same effect should be reflected in a broadening of the vector mesons in dense matter. Since the DLS data show no peak structures which can be attributed to the vector meson masses, the problem to extract information on possible mass shifts is not yet settled. However, the data allow to estimate the order of magnitude of the collision broadening of the vector mesons in heavy ion collisions.

Another question is the role of quantum interference effects. Semi-classical transport models like QMD do not keep track of relative phases between amplitudes but assume generally that decoherent probabilities can be propagated. On the other hand, it has been stressed in several works [27, 30. that, e.g., the interference of the isovector-isoscalar channels, i.e. the so-called $\rho-\omega$ mixing can significantly alter the corresponding dilepton spectra. The $\rho-\omega$ mixing was mainly discussed for the dilepton production in $\pi N$ reactions. Due to the inclusion of excited mesonic states in the resonance decays such interference occurs in our treatment already separately inside each isotopic channel. It is natural to assume that the interference pattern of the mesonic states will be influenced by the presence of surrounding particles. In [35] we discussed decoherence effects which can arise when vector mesons propagate through a hot and dense medium and proposed a simple scheme to model this type of decoherence phenomenon. This discussion is quite general and can be applied, e.g. to the $\rho-\omega$ mixing as a special case. It is assumed that before the first collision with a nucleon or a pion the vector mesons radiate $e^{+} e^{-}$pairs coherently and decoherently afterwards, since the interactions with a heat bath result in macroscopically different final states. As a consequence of charge conservation the coherence must be restored in the soft-dilepton limit. The present model fulfills this boundary condition. The quark counting rules require a destructive interference between the vector mesons entering into the electromagnetic transition form factors of the nucleon resonances. Hence, a break up of the coherence results in an increase of the dilepton yield below the $\rho$-meson peak. This is just the effect observed in the BEVALAC data. Such a quantum decoherence can at least partially resolve the DLS puzzle in heavy ion reactions.

\section{Resonance model and extended VMD}

Usually, the description of the decays of baryonic resonances $R \rightarrow N e^{+} e^{-}$is based on the VMD model in its monopole form, i.e. with only one virtual vector meson $(V=\rho, \omega)$. As the result, the model provides a consistent description of both, radiative $R \rightarrow N \gamma$ and mesonic $R \rightarrow N V$ decays. However, a normalization to the radiative branchings strongly underestimates the mesonic ones [21, 25, 24]. Possible ways to circumvent this inconsistency were proposed in [24, 25]. In [24] a version of the VMD model with vanishing $\rho \gamma$ coupling in the limit of real photons $\left(M^{2}=0\right)$ was used which allows to fit radiative and mesonic decays independently, in 25] an additional direct coupling of the resonances to photons was introduced.

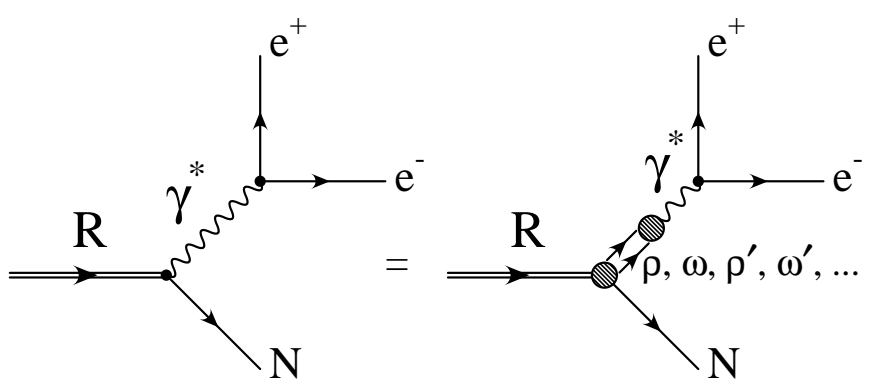

Figure 1: Decay of nuclear resonances to dileptons in the extended VMD model. The $R N \gamma$ transition form factors contain contributions from ground state and excited $\rho$ and $\omega$ mesons.

However, apart from that the standard VMD predicts a $1 / t$ asymptotic behavior for the transition form factors. At the same time the quark counting rules require a stronger suppression at high $t$. A similar problem arises with the $\omega$ Dalitz decay. The $\omega \pi \gamma$ transition form factor shows an asymptotic 
$\sim 1 / t^{2}$ behavior [38]. It has been measured in the time-like region 39] and the data show deviations from the naive one-pole approximation. In [20] it was shown that the inclusion of higher vector meson resonances in the VMD can resolve this problem and provides the correct asymptotics. In [33. the extended VMD (eVMD) model was used to describe the decay of baryonic resonances and in particular to solve the inconsistency between $R N V$ and $R N \gamma$ decay rates. In the eVMD model one assumes that radial excitations $\rho(1250), \rho(1450), \ldots$ can interfere with the ground state $\rho$-meson in radiative processes. Already in the case of the nucleon form factors the standard VMD is not sufficient and radially excited vector mesons $\rho^{\prime}, \rho^{\prime \prime}$... etc. should be added in order to provide a dipole behavior of the Sachs form factors and to describe the experimental data. In view of these facts the present extension of the VMD model is more general than the approach pursued in [24] since it allows not only to describe consistently resonance decays but also other observables like the $\omega$ Dalitz decay or the nucleon form factor. Here we only briefly sketch the basic ideas of the extended vector meson dominance (eVMD) model. In Fig. 1 the resonance decays are schematically displayed for the extended VMD model with excited mesons as intermediate states. The interference between the different meson families plays a crucial role for the behavior of the form factors. Details of the relativistic calculation of the magnetic, electric, and Coulomb transition form factors and the branching ratios of the nucleon resonances can be found in 33 .

The vector meson production cross section in elementary nucleon-nucleon reactions is now given by

$$
\frac{d \sigma(s, M)^{N N \rightarrow N N V}}{d M^{2}}=\sum_{R} \int_{\left(m_{N}+M\right)^{2}}^{\left(\sqrt{s}-m_{N}\right)^{2}} d \mu^{2} \frac{d \sigma(s, \mu)^{N N \rightarrow N R}}{d \mu^{2}} \frac{d B(\mu, M)^{R \rightarrow V N}}{d M^{2}} .
$$

The cross sections for the resonance production are given by

$$
d \sigma(s, \mu)^{N N \rightarrow N R}=\frac{\left|\mathcal{M}_{R}\right|^{2} p_{f}}{16 p_{i} s \pi} d W_{R}(\mu)
$$

with the final c.m. momentum

$$
p_{f}=p^{*}\left(\sqrt{s}, \mu, m_{N}\right)=\frac{\sqrt{\left(s-\left(\mu+m_{N}\right)^{2}\right)\left(s-\left(\mu-m_{N}\right)^{2}\right)}}{2 \sqrt{s}}
$$

and the initial c.m. momentum $p_{i}$. The mass distributions $d W_{R}(\mu)$ of the resonances are usual BreitWigner distributions

$$
d W_{R}(\mu)=\frac{1}{\pi} \frac{\mu \Gamma^{R}(\mu) d \mu^{2}}{\left(\mu^{2}-m_{R}^{2}\right)^{2}+\left(\mu \Gamma_{\text {tot }}^{R}(\mu)\right)^{2}}
$$

where $\mu$ and $m_{R}$ are the running and pole masses, respectively, and $\Gamma(\mu)$ is the mass dependent resonance width. The matrix elements $\mathcal{M}_{R}$ are taken from [40, 41] where they have been adjusted to one and two-pion production data. For the description of the $\rho$ and $\omega$ production in $N N$ and $\pi N$ reactions we consider the same set of resonances which has been used in refs. [21, 34]. It includes only the well established $(4 *)$ resonances listed by the PDG 42 and is smaller than the complete set of resonances included in the QMD model. This set of resonances is, however, sufficient to describe the $N N$ and $\pi N$ vector meson production data.

Fig 2 shows the $\omega$ production in elementary $N N$ reactions. The different cross sections are shown as functions of the excess energy $\epsilon$. As discussed in [34, the resonance model (with a large $N^{*}(1535) N \omega$ coupling) leads to very accurate description of the measured on-shell cross section. It has, however, a very strong off-shell component which fully contributes to the dilepton production. The weak coupling scenario, on the other side, has only small off-shell component but the reproduction of the data is relatively poor in the low energy regime. The parameterization of [43] which has been used in [12, 22 ] is also shown for comparison. 


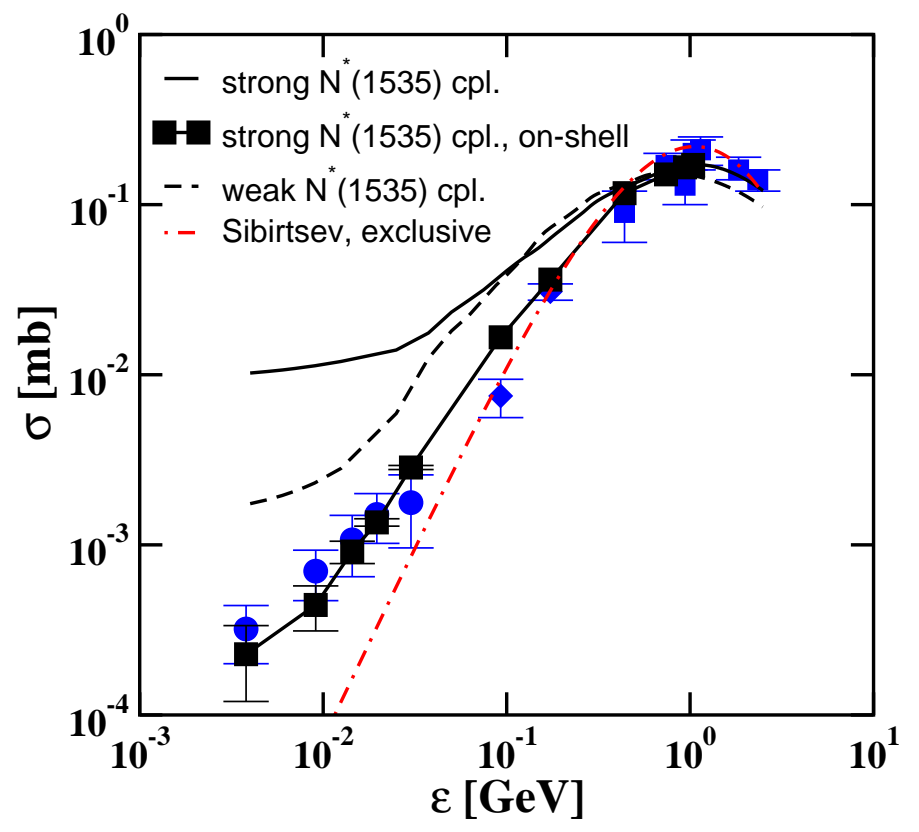

Figure 2: Exclusive $p p \rightarrow p p \omega$ cross section obtained in the resonance model as a function of the excess energy $\epsilon$. The solid curve shows the full cross section including off-shell contributions while the squares show the experimentally detectable on-shell part of the cross section. The dashed curves show the corresponding cross section obtained with weak $N^{*}(1535) N \omega$ coupling. The dot-dashed curve is a parameterization of the exclusive cross section from [43]. Data are taken from [4, 45] and [46, 47].

In ref. 48, the resonances model has been applied to the the production of $\phi$ mesons which, according to the "OZI rule" 49, should be strongly suppressed compared to that of $\omega$ mesons. According to the OZI rule $\phi$ mesons can only be produced due to a small admixture of non-strange light quarks in their wave function. The corresponding mixing angle $\theta_{\text {mix }}$ is equal to $\theta_{\text {mix }} \approx 3.7^{\circ}$. Experimentally, the ratio $R_{\phi / \omega}$ is in $p p \rightarrow p p \phi(\omega)$ reactions, however, known to be one order of magnitude larger than the naive expectation. In ref. [48] it is demonstrated that the experimental data are well reproduced by the present resonance model without introducing additional model parameters.

\section{Dilepton production}

\subsection{Elementary reactions}

Before turning to heavy ion collisions we will consider the dilepton production in elementary reactions. Dilepton spectra in proton-proton and proton deuteron reactions have been measured by the DLS Collaboration in the energy range from $T=1 \div 5 \mathrm{GeV}$ [50. The application of the present model to the dilepton production in $p p$ reactions has in detail been discussed in [21]. We show the corresponding results and the comparison to the DLS data [50] in Fig. 3. The agreement with the available data is generally reasonable, i.e. of similar quality as obtained in previous calculations by Ernst et al. 15 and Bratkovskaja et al. 28. It should be noted that the dilepton yields in $p p$ reactions were obtained with the strong $N^{*}(1535)-N \omega$ decay mode. As in detail discussed in 34 the strong coupling mode is the result of the eVMD fit to the available photo- and meson-production data 33. It leads to sizable contributions from off-shell $\omega$ production around threshold energies which are, however, experimentally not accessible in $p p \rightarrow p p \omega$ measurements. On the other side, these off-shell $\omega$ 's fully contribute to the dilepton yield. The off-shell contributions lead generally to an enhancement of the dilepton yield in the mass region below the $\omega$ peak, in particular at incident energies where the $\omega$ is dominantly produced subthreshold.

The situation becomes more complicated when proton-deuteron reactions are considered. Compared to the $p p$ case one has here two important modifications: First the Fermi motion of the proton and neutron constituents inside the deuteron and secondly, the isotopic relations between the $p p$ and $p n$ 


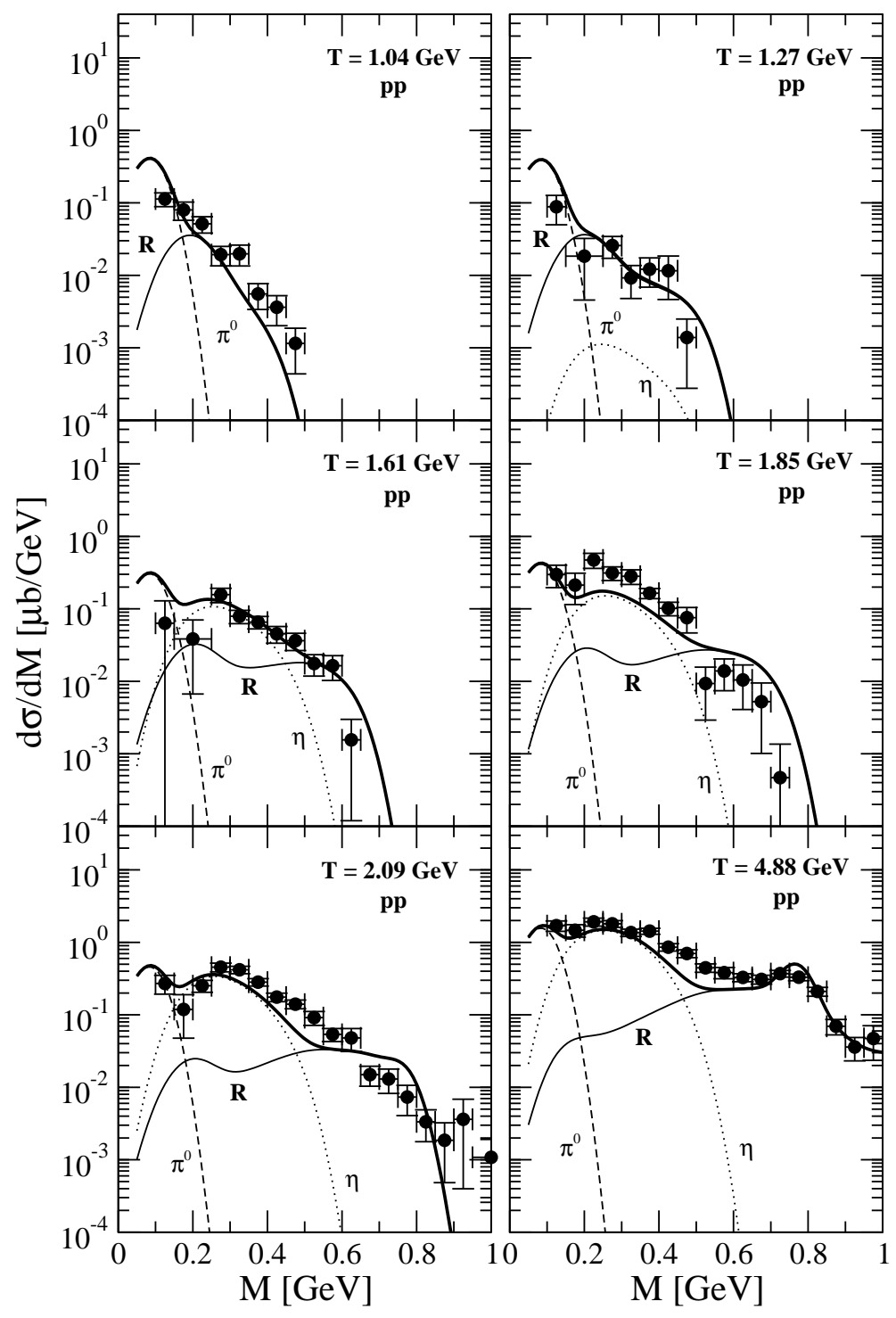

Figure 3: The differential $p p \rightarrow e^{+} e-X$ cross sections at various proton kinetic energies are compared to the DLS data [50].

contributions to the dilepton production. Only few isotopic relations for the meson production are experimentally fixed. Most isospin relations have to be derived from model assumptions. As shown in [35] the present model reproduces the dilepton production in $p d$ collisions at $T=1.61 \div 4.88 \mathrm{GeV}$ rather reasonable. At the two lowest energies $T=1.04 ; 1.27 \mathrm{GeV}$ we underestimate the $p d$ data (probably due to an underestimation of the $\eta$ contribution). At these energies an underestimation which is, however, less pronounced, was also observed in [15. It should be noted that for the $p p$ reactions the present results and those of [15, 28, coincide more or less. In all cases the theoretical calculations reproduce the corresponding DLS data reasonably well. Hence the dilepton production on the deuteron turns out to be rather involved at subthreshold energies due to strong ISI/FSI effects. The $p d$ system is therefore only of limited use to check isospin relations of the applied models. Another important result is the fact that the scenario of large off-shell $\omega$ contributions from the $N^{*}(1535)-N \omega$ decay is consistent with the available $p p$ and $p d$ dilepton data.

\subsection{Heavy ion reactions}

With this input QMD transport calculations for $C+C$ and $C a+C a$ reactions at $1.04 \mathrm{AGeV}$ have been performed [35. First we discuss the results obtained without any additional medium effects concerning the dilepton production. For the nuclear mean field a soft momentum dependent Skyrme 
force $(\mathrm{K}=200 \mathrm{MeV})$ is used which provides also a good description of the subthreshold $K^{+}$production in the considered energy range [51]. The reactions are treated as minimal bias collisions with maximal impact parameters $b_{\max }=5(8)$ fm for $C+C(C a+C a)$.

In Fig. 4 the results are compared to the DLS data. The acceptance filter functions provided by the DLS Collaboration are applied and the results are smeared over the experimental resolution of $\Delta M=35$ $\mathrm{MeV}$. The calculations are performed within the two scenarios discussed in Sec. II, namely a strong $N^{*}(1535)-N \omega$ coupling as implied by the original fit to the available photo-production data 34] and a weaker coupling which can be enforced by a different choice of input parameters. In the first case strong off-shell $\omega$ contributions appear which are also visible in the dilepton spectrum at invariant masses below the $\omega$ peak. In the mass region between $0.4 \div 0.8 \mathrm{GeV}$ the two scenarios yield significantly different results. The rest of the spectrum is practically identical except from the height of the $\omega$ peak itself. As discussed in connection with the elementary cross sections the $\omega$ contribution from the $N^{*}(1535)$ is suppressed at the $\omega$ pole in the strong coupling scenario and thus the total $\omega$ peak is slightly lower. The comparison of the transport calculations with the DLS data is here not completely conclusive: The lighter $C+C$ system would favor the weak $N^{*}(1535)-N \omega$ coupling scenario whereas the $C a+C a$ reactions are better described by the strong coupling. In the low mass region $(M=0.1 \div 0.5 \mathrm{GeV})$

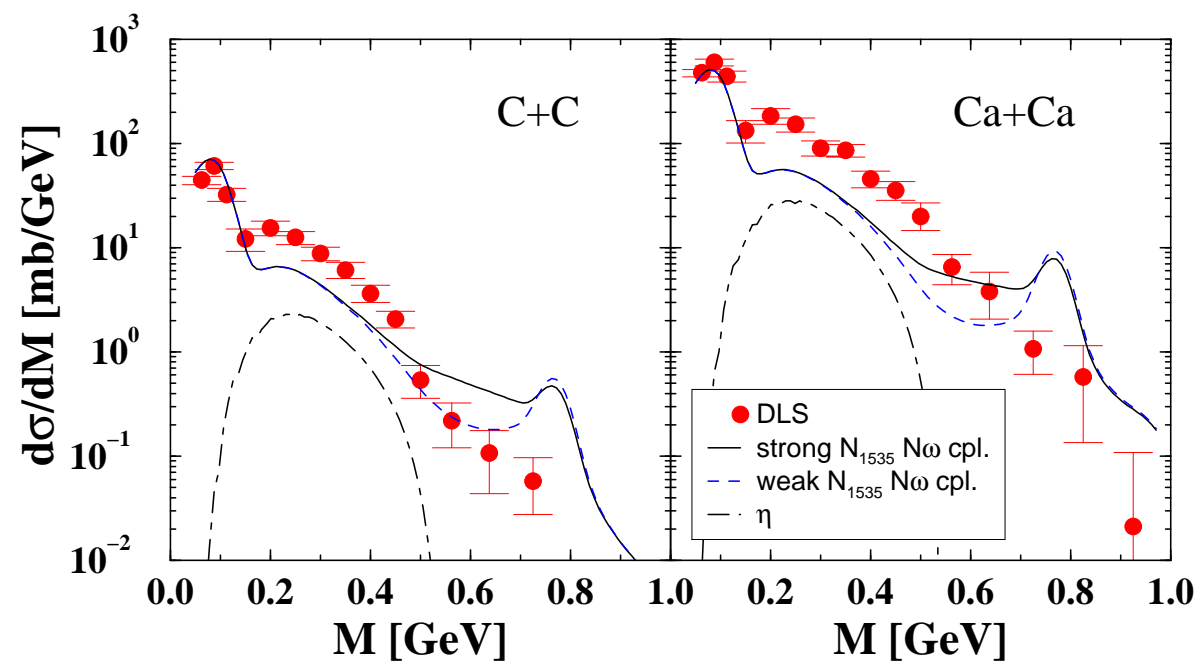

Figure 4: The dilepton spectrum in $C+C$ and $C a+C a$ reactions is compared to the DLS data [14. The calculations are performed with a strong, respectively a weak $N^{*}(1535)-N \omega$ coupling.

we observe an underestimation of the DLS spectra by a factor of $2 \div 3$. Thus in the present approach the underestimation of the DLS data is somewhat smaller than observed in the previous works of [15] and [12]. One reason for this is a larger $\eta$ contribution which is probably due to the iso-spin factor of 6.5 for the $n p \rightarrow n p \eta$ channel (compared to a factor of 2.5 used in [12, 28]). Other differences to the previous treatments [15, 12, are the following: In ref. 12 the vector meson production was described by parameterizations of the $N N$ and $\pi N$ production channels while in the present approach these reactions run solely over the excitation of intermediate nuclear resonances. In [15, 12] only the $\Delta(1232) \rightarrow N e^{+} e^{-}$Dalitz decay has explicitely been included. In addition, the decays of the nucleon resonances into vector mesons were treated till recently in the non-relativistic approximation [28, 24] and usually only one transition form factor was taken into account. From counting the independent helicity amplitudes it is clear that a phenomenologically complete treatment requires three transition form factors for spin $J \geq 3 / 2$ nucleon resonances and two transition form factors for spin- $1 / 2$ resonances. Earlier attempts to derive a complete phenomenological expression for the dilepton decay of the $\Delta(1232)$ were not successful (for a discussion see 32]). Despite of the details which differ in the various transport calculations (we included significantly more decay channels and apply an improved description of the baryonic resonance decays) the present results confirm qualitatively the underestimation of the DLS data at invariant masses below the $\rho / \omega$ peak [15, 12]. 
A deviation to the results of [15] and [12] appears in the vicinity of the $\omega$ peak. Even after averaging over the experimental resolution the present results show a clear peak structure around $0.8 \mathrm{GeV}$ which is absent in 15, 12. However, in 12 absorptive channels (e.g. $N \omega \rightarrow N \pi$ [52]) have been included which lead automatically to a collisional broadening of the in-medium vector meson width. Such a collision broadening is not included in the results shown in Fig. 3.2 but will separately be discussed in the next subsection. With respect to the UrQMD calculations of [15] our approach is in principle similar since vector mesons are produced through the excitation of nuclear resonances. However, in [15] the naive VMD was applied to treat the mesonic decays and the treatment is more qualitative, i.e. couplings were not particularly adjusted in order to describe $\rho$ and $\omega$ cross section as it was done in 33, 34. E.g. in [15] only the $N^{*}(1900) \rightarrow N \omega$ decay mode was taken into account which leads presumably to a significant underestimation of the $N N \rightarrow N N \omega$ cross section.

\section{$3.3 \rho$ - and $\omega$-meson in-medium widths}

In previous studies in-medium spectral functions of the $\rho$ - and $\omega$-mesons were implemented into heavyion codes ab initio [12. At intermediate energies, the sensitivity of the dilepton spectra on the inmedium $\rho$-meson broadening is less pronounced as compared to the $\omega$-meson. Estimates for the collision broadening of the $\rho$ in hadronic matter, i.e. dense nuclear matter or a hot pion gas, predict a collision width which is of the magnitude of the vacuum $\rho$ width. For the $\omega$, on the other hand, the vacuum width is only $8.4 \mathrm{MeV}$ whereas in the medium it is expected to be more than one order of magnitude larger. However, the possibility of a strong in-medium modification of the $\omega$-meson has not attracted much attention in previous studies. The reason is probably due to the fact that the direct information on the $\omega$-meson channels from resonance decays, available through the multichannel $\pi N$ scattering analysis, is quite restricted. The present model provides an unified description of the photo- and electro-production data and of the vector meson and dilepton decays of the nucleon resonances. It provides also a reasonable description of the vector meson and the dilepton production in elementary reactions $(p+p, p+d)$ in the BEVALAC energy range. However, when applied to $A+A$ reactions the model leads to a very strong overestimation of the dilepton yield around the $\omega$-peak which suggests significant medium modifications of the $\omega$ contribution. At low energies, the vector meson production occurs due to decays of nucleon resonances. The in-medium broadening of vector mesons can be understood within the framework of the resonance model. It has qualitatively two major consequences:

1. an increase of the nucleon resonance decay widths $R \rightarrow N V$

2. a decrease of the dilepton branchings $V \rightarrow e^{+} e^{-}$due to the enhanced total vector meson widths.

These two effects are of opposite signs and can be completely described through appropriate modifications of the vector meson propagators entering into the $R N \gamma$ transition form factors $G_{T}\left(M^{2}\right)$. Within the eVMD framework it is sufficient to increase the total widths of the vector mesons. In a less formal way, the effect can be explained as follows: The differential branching

$$
d B(\mu, M)^{R \rightarrow N V}=\frac{d \Gamma_{\mathrm{NV}}^{R}(\mu, M)}{\Gamma_{R}(\mu)}
$$

becomes usually larger with an increasing $V$ meson width which is due to the subthreshold character of the vector meson production through the light nucleon resonances. The dilepton branching of the nucleon resonances

$$
B(\mu)^{R \rightarrow N e^{+} e^{-}} \sim B(\mu)^{R \rightarrow N V} \frac{\Gamma_{V \rightarrow e^{+} e^{-}}}{\Gamma_{V}^{\text {tot }}}
$$

is, on the other hand, inverse proportional to the total vector meson width $\Gamma_{V}^{\text {tot }}$. Hence, an increase of the total width results in a decrease of the dilepton production rate. This effect is particularly 
strong for the $\omega$ since the in-medium $\omega$ width is expected to be more than one order of magnitude greater than in the vacuum [6]. Although the estimates of ref. [6] were based on the standard VMD model which is contradictive with respect to the description of both, the $R N V$ and $R N \gamma$ branchings [21, 24, 25, the qualitative conclusions concerning the magnitude of the in-medium $\omega$ broadening should be valid. A relatively large $\omega$ collision width is not too surprising. According to the $S U(3)$ symmetry the $\omega$ coupling to nucleons is 3 times greater than the $\rho$ coupling. One can therefore expect that at identical kinematical conditions the $N \omega$ cross section will be greater than the $N \rho$ cross section. Since the collision widths are proportional to the cross sections, the same conclusion holds for the collision widths. The $\omega$ contribution is extremely sensitive to the reaction conditions in the course of the heavy ion collisions. While the increase of the total branching $B(\mu)^{R \rightarrow N V}$ depends on kinematical details one can expect that the suppression of the $\omega$ contribution due the enhanced total width $\Gamma_{\omega}^{\text {tot }}$ is an one order of magnitude effect. The latter assumes an additional collision width of $\Gamma_{\rho}^{\text {coll }}=150 \mathrm{MeV}$ which agrees

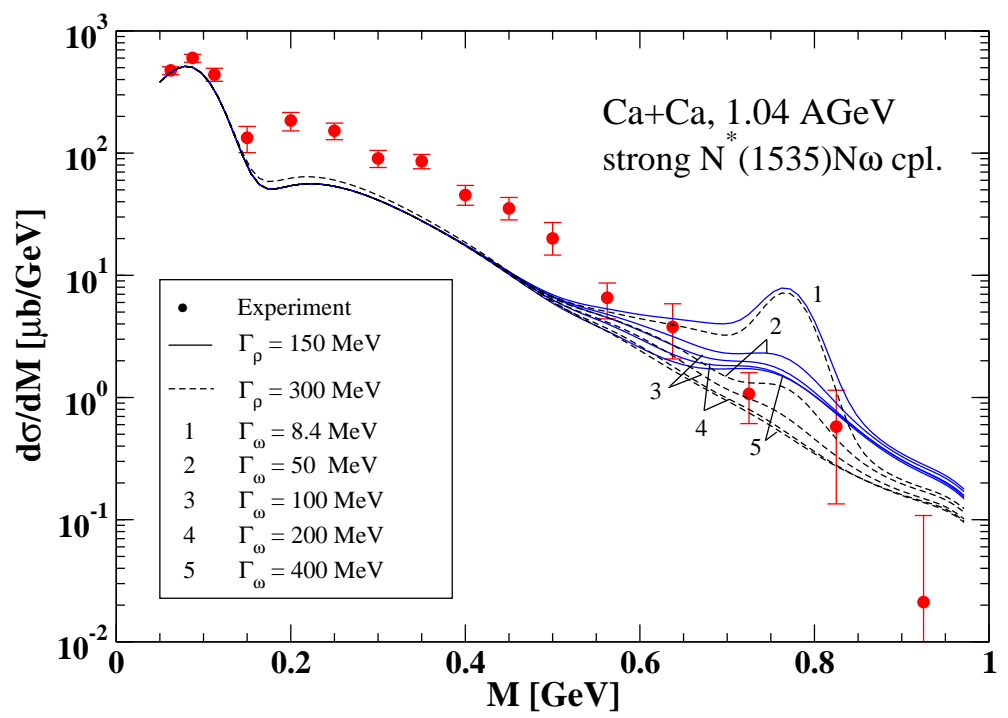

Figure 5: Dilepton spectra in $\mathrm{Ca}+\mathrm{Ca}$ collisions at $1.04 \mathrm{AGeV}$ for different values of the inmedium $\rho$ and $\omega$ widths. The solid curves correspond calculations where the $\rho$ width is kept at its vacuum value of $150 \mathrm{MeV}$ (no collision broadening). The dashed curves correspond to a total $\rho$ width of $300 \mathrm{MeV}$. In both cases the $\omega$ width is varied between $\Gamma_{\omega}^{t o t}=8.4 \div 400 \mathrm{MeV}$.

with the estimates of refs. [4, 5, 6, 7]. In both cases the $\omega$ width is varied between $\Gamma_{\omega}^{\text {tot }}=8.4,50,100$, 200, and $400 \mathrm{MeV}$. As already mentioned, the in-medium $\omega$ broadening is less studied. Thus we cover the possible range of in-medium values by the above parameter set.

First of all, it is important to realize that the region which is sensitive to in-medium modifications of the meson widths is distinct from the mass interval between $0.2 \div 0.6 \mathrm{GeV}$ where the DLS puzzle is observed. This means that the problem to extract in-medium vector meson widths is isolated from the difficulties concerning the theoretical interpretation of the dilepton spectra below the $\rho / \omega$ peak. As expected, the dilepton spectra in the vicinity of the $\rho / \omega$ peak react very sensitive on modifications of the in-medium width. The reproduction of the DLS data requires an in-medium $\omega$ width which lies above $50 \mathrm{MeV}$ for both, strong and weak couplings. As seen from Fig. 5 , the best fits are obtained with $\Gamma_{\rho}^{\mathrm{tot}}=$ $300 \mathrm{MeV}$ and $\Gamma_{\omega}^{\mathrm{tot}}=100 \div 300 \mathrm{MeV}$. With these values we reproduce in the strong $N^{*}(1535) N \omega$ coupling scenario the DLS data points around and $100 \mathrm{MeV}$ below the $\rho / \omega$ peak within error bars. In the weak coupling scenario the DLS data are still slightly underestimated below the peak. However, the situation is not completely conclusive as discussed in detailk in [35. Definite conclusions on the $N^{*}(1535) N \omega$ mode from dilepton yields in heavy ion reactions require more precise data which will be provided by HADES [53. The present estimates can be interpreted as empirical values which are directly extracted from the experiment. The strength of the $\omega$ broadening and the theoretical motivation through Eq. (6) provide confidence for these estimates.

If the average widths are fixed one can, on the other hand, extract an average cross section from the collision broadening condition $\Gamma_{V N}^{\text {coll }}=\left\langle\rho_{B}\right\rangle v \gamma \sigma_{V N}$. The average nuclear density at the vector meson production, respectively at the decay of the corresponding nuclear resonances $R$, is in minimal bias 1 $\mathrm{AGeV} C a+C a$ reactions about 1.5 times the saturation density, i.e. $\left\langle\rho_{B}\right\rangle_{C a+C a}=0.24 \mathrm{fm}^{-3}$ and slightly 
less for $C+C\left(\left\langle\rho_{B}\right\rangle_{C+C}=0.20 \mathrm{fm}^{-3}\right)$. If one assumes now that the vector mesons are produced in an isotropic fireball with a temperature of $T \simeq 80 \mathrm{MeV}$ the extracted collisional width corresponds to an average $\rho N$ cross section of about $\sigma_{\rho N} \simeq 30 \mathrm{mb}$ and $\sigma_{\omega N} \simeq 50 \mathrm{mb}$ for the $\omega\left(\Gamma_{\omega}^{\text {tot }}=200 \mathrm{MeV}\right)$.

\subsection{Decoherence}

In refs. [21, 33], radially excited $\rho$ - and $\omega$-mesons were introduced in the transition form factors $R N \gamma$ to ensure the correct asymptotic behavior of the amplitudes in line with the quark counting rules. Thereby we required a destructive interference between the members of the vector meson families away from the poles of the propagators, i.e. the meson masses. In a dense medium the environment of the vector mesons can be regarded as a heat bath. Usually the different scattering channels of the interaction with a heat bath, i.e. the surrounding nucleons and pions, are summed up decoherently since the various channels acquire large uncorrelated relative phases. In such a case, the coherent contributions to the probability are random and cancel each other. We have in a sense macroscopically different intermediate states which do not interfere since small perturbations result in macroscopically large variations of the relative phases. The interaction of the vector mesons with the surrounding particles should therefore break up the coherence between the corresponding amplitudes for the dilepton production. The break up of the destructive interference results in an increase of the total cross sections at low dilepton masses. In the following we want to investigate if the decoherence effect can explain the enhancement observed in the dilepton spectra at the BEVALAC experiment (DLS puzzle).

In the case of a full decoherence the vector meson contributions to the cross section $N N \rightarrow e^{+} e^{-} X$ which run over nucleon resonances must be summed up decoherently. This leads to the replacement

$$
\left|\sum_{k} \mathcal{M}_{T k}^{( \pm)}\right|^{2} \rightarrow \sum_{k}\left|\mathcal{M}_{T k}^{( \pm)}\right|^{2}
$$

As a consequence, total decoherence will result in an enhancement of the resonance contributions due to the presence of the medium [35].

The decay probability for a resonance at distance $l_{C}$ in the interval $d l_{C}$ equals

$$
d W_{D}\left(l_{D}\right)=e^{-l_{D} / L_{D}} \frac{d l_{D}}{L_{D}} .
$$

The decay length for a resonance with lifetime $T_{D}$ equals $L_{D}=v \gamma T_{D}$, where $T_{D}=1 / \Gamma, \Gamma$ being the total vector meson vacuum width. The collision probability at a distance $l_{C}$ in the interval $d l_{C}$ equals

$$
d W_{C}\left(l_{C}\right)=e^{-l_{C} / L_{C}} \frac{d l_{C}}{L_{C}} .
$$

The collision length $L_{C}$ is defined by the expression

$$
L_{C}=\frac{1}{\rho_{B} \sigma}
$$

where $\sigma$ is the total $V N$ cross section and $\rho_{B}$ is the nuclear density. The meson decay takes place before the first collision provided that $0<l_{D}<l_{C}$, so the probability of the coherent decay equals

$$
w=\int_{0}^{+\infty} \frac{d l_{C}}{L_{C}} e^{-l_{C} / L_{C}} \int_{0}^{l_{C}} \frac{d l_{D}}{L_{D}} e^{-l_{D} / L_{D}}=\frac{L_{C}}{L_{C}+L_{D}} .
$$

All mesons have in general different values $L_{D}$ and $L_{C}$ and thus the coherent decay probabilities are different as well. The collision broadening and the collision length are related through equations

$$
e^{-l_{C} / L_{C}}=e^{-v t / L_{C}}=e^{-\Gamma_{V}^{\mathrm{coll}} t / \gamma} .
$$


Expression (12) provides the the probability that a meson $V$ travels after its creation the length $l_{C}$ through the medium without being scattered by the surrounding hadrons. In Eq.(12), $v$ is velocity and $\gamma$ is the Lorentz factor. The collision length and width are thus related by

$$
v / L_{C}=\Gamma_{V}^{c o l l} / \gamma
$$

The collision length follows from the collisions widths which were extracted in the previous subsection. Since the collision widths are directly extracted from data, the $\rho$ and $\omega$ collision lengths which are necessary in order to determine the probabilities for a coherent dilepton emission can be obtained from (13). The estimates of the collision lengths for radially excited vector mesons are thereby assumed to be the same as for the ground-state vector mesons. The vacuum widths of the radially excited mesons are larger than those of the ground state $\rho$ and $\omega$. As a consequence, the radially excited mesons show a tendency to decay coherently. The decoherence effect is most pronounced for the ground-state $\omega$-meson, since its vacuum width is particularly small. The $\omega$-meson decays in the medium almost fully decoherent, i.e. after its first collision with another hadron. This results in a modification of the $N^{*} \rightarrow N e^{+} e^{-}$decay rates of the $I=1 / 2$ resonances due to the destruction of the interference between the $I=0$ and $I=1$ transition form factors. Since for the considered reactions the matter is isospin symmetric, the break up of the $\rho-\omega$ coherence does not result in a significant change of the dilepton spectra. In this case the isoscalar-isovector interference terms cancel on average. The major effect arises from the break up of the interference between the $\omega$ and its radial excitations.

In [35] the influence of the decoherent summation of the intermediate mesonic states in the transition form factors was in detail investigated. A totally decoherent summation of the mesonic amplitudes in the resonance decays enhances the dilepton yield generally by about a factor of two. In the low mass region this enhancement is able to match the DLS data. However, the scenario of a completely decoherent dilepton emission is rather unrealistic.

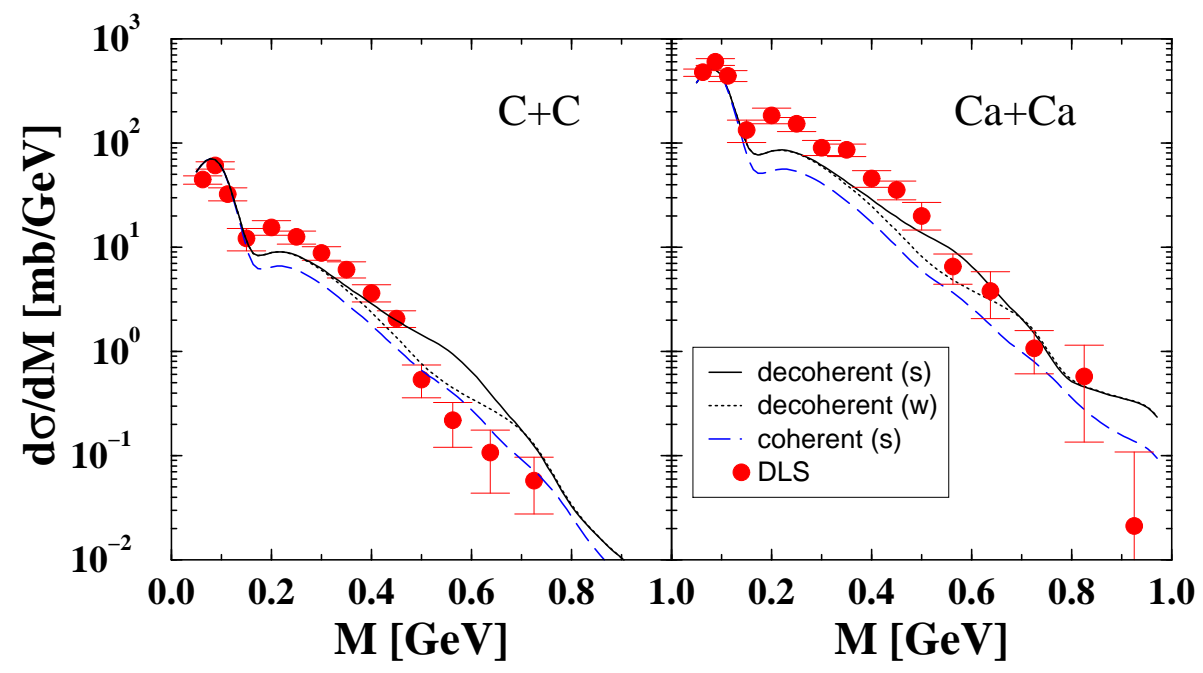

Figure 6: Influence of the microscopically determined decoherent dilepton emission in $C+C$ and $C a+C a$ reactions. A strong (s), respectively, weak (w) $N^{*}(1535)-N \omega$ coupling is used. For comparison also the coherent case (s) is shown.

In a realistic calculation shown in Fig. 6 the probabilities for coherent/decoherent dilepton emission are determined microscopically as outlined above, i.e. by the use of Eqs. (11] 13). These use the 'optimal' values for the in-medium widths of $\Gamma_{\rho}^{\text {coll }}=150, \Gamma_{\omega}^{\text {coll }}=200 \mathrm{MeV}$. The low mass dilepton yield is now enhanced by about $50 \%$ by the decoherence effect which is, however, still too less to describe the DLS data. The interplay between the two in-medium effects, i.e. the collisional broadening and the decoherent dilepton emission is more complex. Decoherence leads also to an enhancement of the dilepton yield in the mass region between $0.4 \div 0.7 \mathrm{GeV}$. Since the main decoherence effect occurs through the broken interference of the $\omega$ with its excited states, it is most pronounced in the dilepton contribution which stems from the $N^{*}$ resonance decays. This explains the difference between the two 
calculations assuming a strong/weak $N^{*}(1535) N \omega$ coupling in the mass range where possible off-shell $\omega$ contributions are now enhanced (strong coupling). However, definite conclusions on the strength of the $N^{*}(1535) N \omega$ coupling are still difficult to make at the present data situation. For the strong coupling the $C a+C a$ system is in agreement within error bars with the DLS data whereas in the lighter $C+C$ system the data are now overestimated and would favor the weak coupling. In both cases the agreement with the data is significantly improved in the low mass region. However, the considered decoherence effects are not completely sufficient in order to solve the DLS puzzle. The reason is that the microscopic determination of the decoherence probability favors the break up of the coherence between the $\omega$ and its excited states in the $N^{*}$ decays rather than the break up between the $\rho$ and its excited states in the $\Delta$ decays. The latter resonances are, however, those which contribute to most extent at low invariant masses.

\section{Conclusion}

In the present work we provided a systematic description of vector meson and dilepton production in elementary $N N$ and $\pi N$ as well as in $A+A$ reactions. The reactions dynamics of the heavy ion collisions is described by the QMD transport model which was extended for the inclusion of nucleon resonances with masses up to $2 \mathrm{GeV}$. The vector meson production in elementary reactions is described through excitations of nuclear resonances within the framework of an extended VMD model. The model parameters were fixed utilizing electro- and photo-production data as well as $\pi N$ scattering analysis. Available data on the $\rho$ and $\omega$ production in $p+p$ and $\pi+N$ reactions are well reproduced. The same holds for the dilepton production in elementary $p+p$ and $p+d$ reactions.

The situation becomes different turning to heavy ion collisions: In $C+C$ and $C a+C a$ reactions we observe in two distinct kinematical regions significant deviations from the dilepton yields measured by the DLS Collaboration. At small invariant masses the experimental data are strongly underestimated which confirms the observations made by other groups. Although accounting for the experimental resolution we observe further a clear structure of the $\rho / \omega$ peak which is not present in the data. Both features imply the investigation of further medium effects.

The collisional broadening of the vector mesons suppresses the $\rho / \omega$ peak in the dilepton spectra. This allows to extract empirical values for the in-medium widths of the vector mesons. From the reproduction of the DLS data the following estimates for the collision widths $\Gamma_{\rho}^{\text {coll }}=150 \mathrm{MeV}$ and $\Gamma_{\omega}^{\text {coll }}=100-300 \mathrm{MeV}$ can be made. The in-medium values correspond to an average nuclear density of about $1.5 \rho_{0}$. HADES will certainly help to constrain these values with higher precision.

The second medium effect discussed here concerns the problem of quantum interference. Semiclassical transport models like QMD do generally not account for interference effects, i.e. they propagate probabilities rather than amplitudes and assume that relative phases cancel the interference on average. However, interference effects can play an important role for the dilepton production. In the present model the decay of nuclear resonances which is the dominant source for the dilepton yield, requires the destructive interference of intermediate $\rho$ and $\omega$ mesons with their excited states. The interference can at least partially be destroyed by the presence of the medium which leads to an enhancement of the corresponding dilepton yield. We proposed a scheme to treat the decoherence in the medium on a microscopic level. The account for decoherence improves the agreement with the DLS data in the low mass region. However, the magnitude of this effect is not sufficient to resolve the DLS puzzle completely.

\section{References}

[1] G.E. Brown and M. Rho, Phys. Rev. Lett. 66 (1991) 2720 ; ibid., Phys. Rep. 269 (1996) 333 
[2] T. Hatsuda and S.H. Lee, Phys. Rev. C 46 (1992) R34;

Y. Koike, Phys. Rev. C 51 (1995) 1488 ;

T. Hatsuda, S.H. Lee, H. Shiomi, Phys. Rev. C 52 (1992) 3364 ;

S. Leupold, Phys. Rev. C 64 (2001) 015202 ;

S. Zschoke, O.P. Pavlenko, B. Kämpfer, Eur. J. Phys. A 15 (2002) 529

[3] A. Bhattacharyya, S.K. Ghosh and S.C. Phatak, Phys. Rev. C 60 (1999) 044903

[4] F. Klingl, N. Kaiser, W. Weise, Nucl. Phys. A 624 (1997) 527 ;

F. Klingl, W. Weise, Eur. J. Phys. A 4 (1999) 225

[5] V.L. Eletsky, B.L. Ioffe, Phys. Rev. Lett. 78 (1997) 1010

[6] V.L. Eletsky, M. Belkasem, P.J. Ellis, J.I. Kapusta, Phys. Rev. C 64 (2001) 035202

[7] L.A. Kondratyuk et al., Phys. Rev. C 58 (1998) 1078

[8] G. Agakichiev et al., Phys. Rev. Lett. 75 (1995) 1272 ; A. Drees, Nucl. Phys. A 610 (1996) 536c

[9] M. Masera, Nucl. Phys. A 590 (1995) 93c

[10] W. Cassing, W. Ehehalt, C.M. Ko, Phys. Lett. B 363 (1995) 35 ;

G.Q. Li, C.M. Ko, G.E. Brown, Nucl. Phys. A 606 (1996) 568 ;

C.M. Hung, E.V. Shuryak, Phys. Rev. C 56 (1997) 453453

[11] M. Urban, M. Buballa, R. Rapp, J. Wambach, Nucl. Phys. A 641 (1998) 433 ;

R. Rapp and J. Wambach, Adv. Nucl. Phys. 25 (2000) 1

[12] E.L. Bratkovskaya, W. Cassing, R. Rapp, J. Wambach, Nucl. Phys. A 634 (1998) 168

[13] R. Schneider and W. Weise, JournalEur. J. Phys. A93572000

[14] R.J. Porter et al. [DLS Coll.], Phys. Rev. Lett. 79 (1997) 1229

[15] C. Ernst, S.A. Bass, M. Belkacem, H. Stocker and W. Greiner, Phys. Rev. C 58 (1998) 447

[16] E.L. Bratkovskaya and C.M. Ko, Phys. Lett. B 445 (1999) 265

[17] T. Inoue, E. Oset, Nucl. Phys. A 710 (2002) 354

[18] K. Ozawa et al., Phys. Rev. Lett. 86 (2001) 5019

[19] E.L. Bratkovskaya, Phys. Lett. B 529 (2002) 26

[20] A. Faessler, C. Fuchs and M.I. Krivoruchenko, Phys. Rev. C 61 (2000) 035206

[21] A. Faessler, C. Fuchs, M.I. Krivoruchenko and B.V. Martemianov, J. Phys. G 29 (2003) 603

[22] W. Cassing, E.L. Bratkovskaya, M. Effenberger and U. Mosel, Nucl. Phys. A 653 (1999) 301

[23] C. Song and V. Koch, Phys. Rev. C 54 (1995) 1903

[24] B. Friman, H.J. Pirner, Nucl. Phys. A 617 (1997) 496

[25] M. Post, U. Mosel, Nucl. Phys. A A688 (2001) 808

[26] A.I. Titov, B. Kämpfer, Eur. J. Phys. A 12 (2001) 217 
[27] M. Zetenyi, Gy. Wolf, Heavy Ion Phys. 17 (2003) 27

[28] E.L. Bratkovskaya, W. Cassing and U. Mosel, Nucl. Phys. A 686 (2001) 568

[29] E.L. Bratkovskaya, Nucl. Phys. A A696 (2) ( 001) 761;

M. Effenberger, E.L. Bratkovskaya, W. Cassing, U. Mosel, Phys. Rev. C C60 (1) ( 999) 027601.

[30] M.F.M. Lutz, B. Friman, M. Soyeur, Nucl. Phys. A A713 (2) ( 003) 97.

[31] G. Penner, U. Mosel, Phys. Rev. C 66 (2002) 055211 ; Phys. Rev. C 66 (2002) 055212

[32] M.I. Krivoruchenko and Amand Faessler, Phys. Rev. D 65 (2002) 017502

[33] M.I. Krivoruchenko, B.V. Martemyanov, A. Faessler, and C. Fuchs, Ann. Phys. (N. Y.) 296 (2002) 299

[34] C. Fuchs, M.I. Krivoruchenko, H. Yadav, A. Faessler, B.V. Martemyanov, K. Shekther, Phys. Rev. C 67 (2003) 025202

[35] K. Shekhter, C. Fuchs, Amand Faessler, M.I. Krivoruchenko, B.V. Martemyanov, Phys. Rev. C 68 (2003) 014904

[36] N. Bianchi et al., Phys. Lett. B 309 (1993) 5 ;

Phys. Lett. B 325 (1994) 333

[37] L. A. Kondratyuk, M. I. Krivoruchenko, N. Bianchi, E. D. Sanctis and V. Muccifora, Nucl. Phys. A 579 (1994) 453

[38] A.I. Vainstein and V.I. Zakharov, Phys. Lett. B 72 (1978) 368

[39] L.G. Landsberg, Phys. Rep. 128 (1985) 301

[40] S. Teis, W. Cassing, M. Effenberger, A. Hombach, U. Mosel, and Gy. Wolf, Z. Phys. A A356 (1997) 421

[41] S.A. Bass et al., Prog. Part. Nucl. Phys. A 41 (1998) 225

[42] Particle Data Group, Phys. Rev. D 54 (1) ( 996).

[43] A. Sibirtsev, W. Cassing, U. Mosel, Z. Phys. A 358 (1997) 357

[44] F. Hibou et al., Phys. Rev. Lett. 83 (1999) 492

[45] COSY-TOF Collaboration, Phys. Lett. B 522 (2001) 16

[46] F. Balestra et al. [DISTO Coll.], Phys. Rev. C 63 (2001) 024004

[47] V. Flaminio et al., CERN-HERA 84-10 (1984).

[48] Amand Faessler, C. Fuchs, M.I. Krivoruchenko, B.V. Martemyanov, Phys. Rev. C 68 (2003) 068201

[49] S. Okubo, Phys. Lett. B 5 (1963) 165 ;

G. Zweig, CERN Report No.8419/TH, 412 (1964);

I. Iizuka, Prog. Theor. Phys. 37 (1966) 21

[50] W.K. Wilson et al. [DLS Coll.], Phys. Rev. C 57 (1998) 1865 
[51] C. Fuchs, Amand Faessler, E. Zabrodin, Y. M. Zheng, Phys. Rev. Lett. 86 (2001) 1974

[52] E.L. Bratkovskaya and W. Cassing, Phys. Rep. 308 (1999) 65

[53] J. Friese [HADES Coll.], Nucl. Phys. A 654 (1999) 1017c 